\title{
Microwave Staring Correlated Imaging Method Based on Bistatic Radar
}

\author{
Yuanyue Guo ${ }^{1}$, Lin Chen ${ }^{2}$ and Dongjin Wang ${ }^{1+}$ \\ ${ }^{1}$ Key Laboratory of Electromagnetic Space Information, Chinese Academy of Sciences \\ ${ }^{2}$ University of Science and Technology of China
}

\begin{abstract}
Microwave staring correlated imaging based on the temporal-spatial stochastic radiation field is a radar system that can not only derive the target image without motion limitation but also obtain higher imaging resolution beyond the aperture limitation. The basic principle of such a radar imaging system is to employ the temporal-spatial stochastic radiation field, which is produced by a multi-transmitter configuration, to make scatters located at different positions reflect independent echo from each other, and then to derive the target image based on the prior knowledge of this radiation field. Furthermore, the more stochastic the radiation field is, the better imaging quality could be derived. This paper illustrates an approach to improve the spatial stochastic degree of radiation field using bistatic radar. By constructing the mathematic model, the principle of this proposal are analyzed in detail in this paper. Finally a stimulation is carried out to verify that this proposal is obviously useful to improve the stochastic characteristics of radiation field and the imaging performance.
\end{abstract}

Keywords: microwave staring correlated imaging, stochastic radiation field, bistatic radar.

\section{Introduction}

When traditional real aperture radar is used for staring imaging to a static target, its azimuth resolution is limited by its antenna aperture, resulting in low imaging resolution. Though synthetic aperture radar (SAR) and inverse synthetic aperture radar (ISAR) are able to obtain high azimuth resolution, they are dependent on the relative motion between radar and target [1]. Microwave staring correlated imaging based on temporalspatial stochastic radiation field is a new imaging method which could obtain high-resolution without relative motion. Its basic principle is employing a temporal-spatial stochastic radiation field, generated by a radar array, to make target located at different positions reflect independent echoes at different moments. Then, we use the echo samples and the known radiation field to extract scattering characteristic of the target by means of correlation processing algorithm. Consequently, the more stochastic the radiation field is, the more information about the target will be extracted [2]. Thus, the stochastic degree of radiation field plays a significant role on imaging effect.

Currently, researchers have raised some ideas on improving the stochastic degree of radiation field such as increasing the amount of transmitting units, enlarging the area of radar array, increasing signal bandwidth and so on [3]. Compared with uniform distribution of transmitting units on the radar array plane, using a genetic algorithm to optimize the layout of transmitters could increase the diversity of radiation field [4]. Jun Li suggested to spin the radar array to raise the rate of radiation field change [5]. Above studies focus on the optimization of the emission source as well as the radar receiver being put on the center of radar array indiscreetly, namely monostatic radar system. Therefore, this paper focus on improving the stochastic degree of radiation field by adopting bistatic radar system.

\footnotetext{
+ Corresponding author. Tel.: +86-13866135598; fax: +(86-551-63603942).

E-mail address: yuanyueg@ustc.edu.cn.
} 
This paper is organized as follow. Section II briefly gives the fundamental analysis of microwave staring correlated imaging and illustrates the relationship between the transceiver angle and the stochastic degree of radiation field in detail. Along with stimulation results, the effectiveness and performance of this proposal is analyzed in section III. Section IV concludes the work.

\section{Analysis of Microwave Staring Correlated Imaging Based on Bistatic Radar}

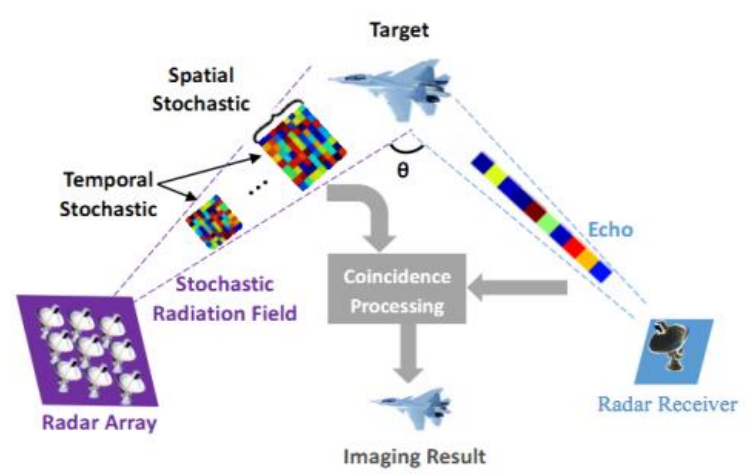

(a)

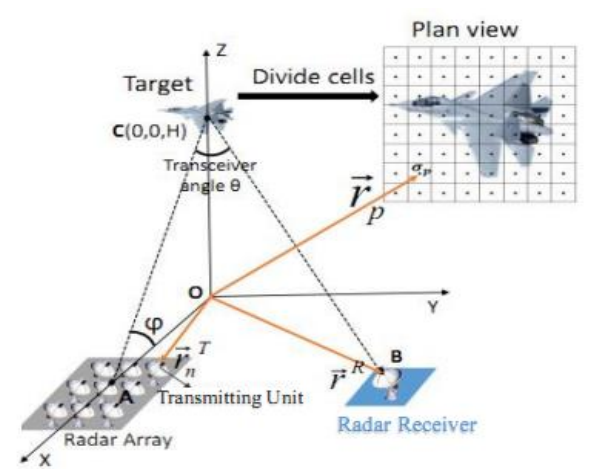

(b)

Fig. 1: Scene of staring correlated imaging based on bistatic radar.

\subsection{Microwave staring correlated imaging model based on bistatic radar}

Microwave staring correlated imaging system based on bistatic radar are depicted in Fig. 1(a). The radar array is composed of $N$ transmitting units and it is away from the radar receiver. Meanwhile, The radar array and the radar receiver form a wide transceiver angle $\theta$ relative to the target region, defined as

$$
\cos \theta=\frac{\left(\vec{r}^{T}-\vec{r}^{O}\right) \cdot\left(\vec{r}^{R}-\vec{r}^{O}\right)}{\left|\vec{r}^{T}-\vec{r}^{O}\right| \cdot\left|\vec{r}^{R}-\vec{r}^{O}\right|}
$$

where $\vec{r}^{T}, \vec{r}^{R}$ and $\vec{r}^{O}$ is the center position of the radar array, the radar receiver and the target region, respectively. In order to generate time-varying signals which are uncorrelated from different units, transmitting unit emits $M$ randomly pulsed signal with random amplitude modulation, random frequency modulation and/or random phase modulation. Hereinto, signal from $n$-th unit is

$$
s\left(\vec{r}_{n}^{T}, t\right)=\sum_{m=1}^{M} A_{n, m} r e c t\left(\frac{t-m T_{t}}{T_{d}}\right) \exp \left\{j 2 \pi f_{n, m}\left(t-m T_{t}\right)+j \theta_{n, m}\right\}
$$

where $\vec{r}_{n}^{T}$ is the position of $n$-th transmitting unit, ${ }^{n=1,2, \cdots, N} . T_{t}$ is the pulse period and $T_{d}$ is the pulse width. $A_{n, m}$ is the amplitude, $f_{n, m}$ is the frequency and $\theta_{n, m}$ is the phase of the $m$-th pulse from the $n$-th transmitting unit, respectively. Transformed by transmitting antenna, signals become incident radiation. Then, after the first propagation in free-space, incident radiation in the target region is

$$
E^{i n c}(\vec{r}, t)=\sum_{n=1}^{N} \frac{F_{n}\left(\hat{R}_{n}\right)}{4 \pi R_{n}} S\left(\vec{r}_{n}^{T}, t-\frac{R_{n}}{c}\right)
$$

where ${ }^{t}$ is observation time, $\vec{r}$ is arbitrary point within the target region $S(\vec{r})$ and its distance from the $n$-th transmitting unit is $R_{n}=\left|\vec{r}-\vec{r}_{n}^{T}\right|$, its unit vector toward the $n$-th transmitting unit is $\hat{R}_{n}=\left(\vec{r}-\vec{r}_{n}^{T}\right) /\left|\vec{r}-\vec{r}_{n}^{T}\right|$. $F_{n}\left(\hat{R}_{n}\right)$ is the radiation pattern of the $n$-th transmitting antenna and ${ }^{c}$ is the speed of light. After reflected by the target and a second propagation, radiation field reaches the radar receiver, namely modified radiation field and expressed as

$$
E^{r a d}\left(\vec{r}, \vec{r}^{R}, t\right)=\sum_{n=1}^{N} \frac{F_{n}\left(\hat{R}_{n}\right) F_{r}\left(\hat{R}_{r}\right)}{16 \pi^{2} R_{n} R_{r}} S\left(\vec{r}_{n}^{T}, t-\frac{R_{n}+R_{r}}{c}\right)
$$


where $R_{r}=\left|\vec{r}^{R}-\vec{r}\right|, \hat{R}_{r}=\left(\vec{r}^{R}-\vec{r}\right) /\left|\vec{r}^{R}-\vec{r}\right|$ and $F_{r}\left(\hat{R}_{r}\right)$ is the radiation pattern of the receiving antenna.

Besides, the stochastic degree of radiation field can be evaluated by correlation function of $E^{r a d}\left(\vec{r}, \vec{r}^{R}, t\right)$, denoted as (5).

$$
\mathrm{R}\left(\vec{r}_{p}, \vec{r}_{q}\right)=\int E^{r a d}\left(\vec{r}_{p}, \vec{r}^{R}, t\right) E^{r a d^{*}}\left(\vec{r}_{q}, \vec{r}^{R}, t\right) d t
$$

and for an ideal temporal-spatial stochastic radiation field, $\mathrm{R}\left(\vec{r}_{p}, \vec{r}_{q}\right)=\delta\left(\vec{r}_{p}-\vec{r}_{q}\right), \forall \vec{r}_{p}, \vec{r}_{q} \in S(\vec{r})$.

Furthermore, according to electromagnetic scattering theory, the echo received by the radar receiver is

$$
S_{r}\left(\vec{r}^{R}, t\right)=\iint_{S(\vec{r})} \sum_{n=1}^{N} \frac{F_{n}\left(\hat{R}_{n}\right) F_{r}\left(\hat{R}_{r}\right)}{(4 \pi)^{2} R_{n} R_{r}} S\left(\vec{r}_{n}^{T}, t-\frac{R_{n}+R_{r}}{c}\right) \cdot \sigma(\vec{r}) d \vec{r}=\iint_{S(\vec{r})} E^{r a d}\left(\vec{r}, \vec{r}^{R}, t\right) \cdot \sigma(\vec{r}) d \vec{r}
$$

where $\sigma(\vec{r})$ is the scattering coefficient of the target located at $\vec{r}$. Then, the time domain is discretized as $t=\left[\begin{array}{llll}t_{1} & t_{2} & \cdots & t_{M}\end{array}\right]$ and the target region compose a vector $\boldsymbol{\sigma}(\vec{r})=\left[\begin{array}{llll}\sigma\left(\vec{r}_{1}\right) & \sigma\left(\vec{r}_{2}\right) & \cdots & \sigma\left(\vec{r}_{L}\right)\end{array}\right]^{T}$ if it is discretized to $L$ grid cells. Hence, above equation could be written as (7) in matrix equation form.

$$
\left.\begin{array}{c}
\mathbf{S}_{\mathbf{r}}=\mathbf{E}^{\mathrm{rad}} \cdot \boldsymbol{\sigma} \\
S_{r}\left(\vec{r}^{R}, t_{1}\right) \\
\vdots \\
S_{r}\left(\vec{r}^{R}, t_{M}\right)
\end{array}\right]=\left[\begin{array}{ccc}
E^{\text {rad }}\left(\vec{r}_{1}, \vec{r}^{R}, t_{1}\right) & \cdots & E^{r a d}\left(\vec{r}_{L}, \vec{r}^{R}, t_{1}\right) \\
\vdots & \ddots & \vdots \\
E^{\text {rad }}\left(\vec{r}_{1}, \vec{r}^{R}, t_{M}\right) & \cdots & E^{\text {rad }}\left(\vec{r}_{L}, \vec{r}^{R}, t_{M}\right)
\end{array}\right] \cdot\left[\begin{array}{c}
\sigma\left(\vec{r}_{1}\right) \\
\vdots \\
\sigma\left(\vec{r}_{L}\right)
\end{array}\right]
$$

Finally, target reconstruction can be achieved by the correlation processing algorithm using the known radiation field $\mathbf{E}^{\text {rad }}$ and the echo samples $\mathbf{S}_{\mathbf{r}}$.

$$
\hat{\boldsymbol{\sigma}}(\vec{r})=\wp\left[\mathbf{E}^{\mathrm{rad}}, \mathbf{S}_{\mathbf{r}}\right]
$$

There into, $\wp(\cdot)$ indicates the first-order correlated operator. Apparently, equation (8) has unique solution and the scattering coefficient can be calculated as long as the matrix $\mathbf{E}^{\text {rad }}$ is nonsingular. Therefore, the matrix rank of $\mathbf{E}^{\text {rad }}$ presents the imaging ability and higher rank indicates a more ideal radiation field and higher imaging resolution.

\subsection{Analysis of the stochastic degree of radiation field}

For sake of concision, the transceiver angle's influence on the stochastic degree of radiation field is demonstrated in below specific scene. An X-Y-Z coordinate system is located beneath the target region, as depicted in Fig. 1(b). The height of target is $H$ and the radar array illuminates the target with an elevation angle ${ }^{\varphi} \cdot A(H / \tan \varphi, 0,0)$ and $B(H / \tan \varphi, H \tan \theta / \sin \varphi, 0)$ are the coordinates of radar array center and radar receiver, respectively. Assuming point $C^{\prime}(x, y, H)$ is in the target region, its propagation distance is $L(x, y)=\left|A C^{\prime}\right|+\left|B C^{\prime}\right|$, which can be approximated to (9) using 2nd-Order-Taylor's approximation.

$$
L(x, y)=\frac{H}{\sin \varphi}+\frac{H}{\sin \varphi \cos \theta}-2 x \cos \varphi-y \sin \theta
$$

Thus, path difference between two adjacent points, $p(0,0, H)$ and $q(\Delta x, \Delta y, H)(\Delta x, \Delta y>0)$, can be briefly expressed as (10). Obviously, path difference $\Delta L$ increases as transceiver angle $\theta$ enlarges when $0^{\circ}<\theta<90^{\circ}$.

$$
\Delta L_{p q}(\theta)=L_{p}(0,0)-L_{q}(\Delta x, \Delta y)=2 \Delta x \cos \varphi+\Delta y \sin \theta
$$

On the other hand, since all signals from different transmitting units are orthogonal in different instant, the correlation of $E^{\text {rad }}\left(\vec{r}, \vec{r}^{R}, t\right)$ can be approximated to 


$$
\mathrm{R}\left(\vec{r}_{p}, \vec{r}_{q}\right) \approx M \sum_{n=1}^{N} \frac{F_{n}\left(\hat{R}_{n p}\right) F_{r}\left(\hat{R}_{r p}\right) F_{n}\left(\hat{R}_{n q}\right) F_{r}\left(\hat{R}_{r q}\right)}{256 \pi^{4} R_{n p} R_{r p} R_{n q} R_{r q}} \cdot \cos \left(2 \pi \frac{\Delta \mathrm{L}_{p q}}{\lambda_{n}}\right) \propto \cos \left(2 \pi \frac{\Delta \mathrm{L}_{p q}}{\lambda}\right)
$$

where $\lambda$ is the wavelength of signals. Under the circumstances of using microwave and for two adjacent points, it shows that $\mathrm{R}\left(\vec{r}_{p}, \vec{r}_{q}\right)$ will reduce rapidly as path difference $\Delta \mathrm{L}_{p q}$ increases. In conclusion, the stochastic degree of radiation field is better by using bistatic radar and enlarging the transceiver angle $\theta$.

\section{Simulation and Analysis}

As shown in Fig. 1(b), the elevation angle $\varphi=45^{\circ}$ and the height of target region $H$ is 50 meters. The coordinates of radar array center and the radar receiver are $A(50,0,0)$ and $B(50,50 \sqrt{2} \tan \theta, 0)$, respectively. The main system parameters are showed in Table I .

TABLE I: SIMULATION PARAMETERSABLE

\begin{tabular}{|c|l|l|c|l|l|}
\hline No & Simulation Parameters & Value & No & Simulation Parameters & Value \\
\hline 1 & Number of transmitting units N & 25 & 5 & Area of the target region & $10 \mathrm{~m}^{*} 10 \mathrm{~m}$ \\
\hline 2 & Area of the radar array & $2 \mathrm{~m} * 2 \mathrm{~m}$ & 6 & Grid gap & $0.25 \mathrm{~m} * 0.25 \mathrm{~m}$ \\
\hline 3 & Signal frequency & $9.5 \mathrm{GHz}$ & 7 & Number of the grid cell L & 1600 \\
\hline 4 & Bandwidth & $1 \mathrm{GHz}$ & 8 & Sampling number M & 2000 \\
\hline
\end{tabular}

Firstly, the correlation function of $E^{\text {rad }}\left(\vec{r}, \vec{r}^{R}, t\right)$, as defined in formula (5), is given in Fig. 2(a)-(d). It can be seen that with the increase of the transceiver angle $\theta$, the radiation field has better correlation function with narrower main-lobe and lower side-lobe, namely better stochastic degree.

Secondly, as shown in Fig. 2(e), the relationship between the matrix rank of $\mathbf{E}^{\text {rad }}$ and the transceiver angle $\theta$ indicates that rank, standing for stochastic degree of the radiation field, increase as $\theta$ enlarges.

Finally, imaging results are provided with different transceiver angle $\theta$ to verify the effectiveness of this proposal. In Fig. 2(g), the image is quite fuzzy and hard to distinguish. It looks better but the head of the plane is still fuzzy when $\theta=30^{\circ}$. Keeping enlarging $\theta$ and when it gets to $45^{\circ}$, the image becomes very clear. Under circumstance of above simulation conditions, the azimuth resolution of traditional real aperture radar is 0.75 meters, which reveals that this method can obtain 3 times resolution than traditional one.

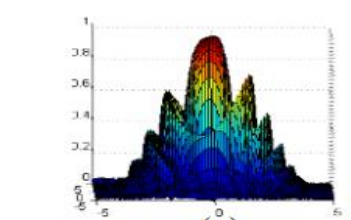

(a)

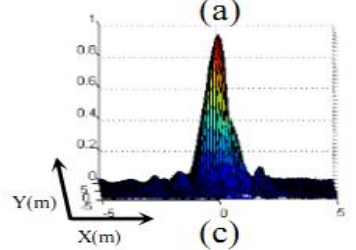

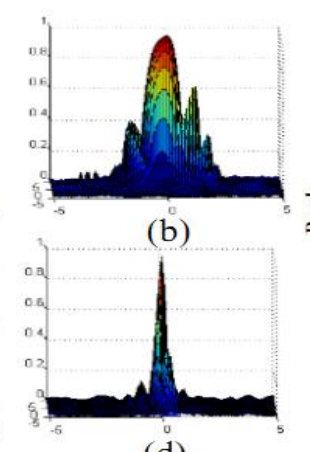

(d)

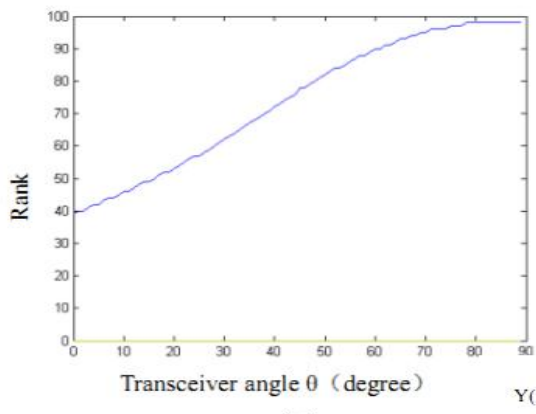

(e)

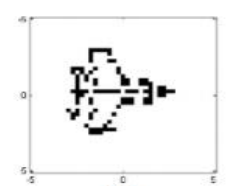

(f)

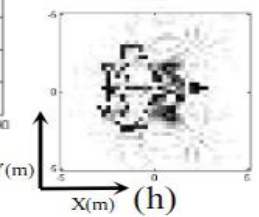

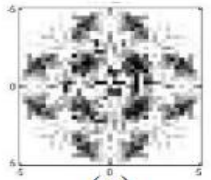

(g)

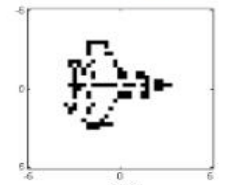

(i)

(a) correlation function of radiation field with different transceiver angle $\theta$ when $\theta=0, \theta=15^{\circ}, \theta=30$ $\theta=45^{\circ}$, respectively. (e) The relationship between transceiver angle $\theta$ and matrix rank of $\mathbf{E}^{\text {rdd }}$. (f) Target scene. (g)-(i) Imaging result with different transceiver angle $\theta$ when $\theta=0^{\circ}, \theta=30^{\circ}, \theta=45^{\circ}$, respectively.

\section{Conclusion}

The key to get higher azimuth resolution of microwave staring correlated imaging is to construct an ideal stochastic radiation field. This paper illustrates an approach to improve the stochastic degree of radiation 
field by using bistatic radar system. It is verified that the stochastic degree and the imaging quality get improvement by adopting this proposal and enlarging the transceiver angle.

\section{Acknowledgements}

This work was supported by the Hi-Tech Research and Development Program of China (Grant Project No.2013AA122903).

\section{References}

[1] D. A. Ausherman, A. Kozma, J. L. Walker, H. M. Jones, and E. C. Pog-gio, "Developments in radar imaging," IEEE Trans. Aerosp. Electron.Syst. vol. AES-20, no. 4, pp. 363-400, Jul. 1984.

[2] Li D, Li X, Qin Y, et al. Radar coincidence imaging: An instantaneous imaging technique with stochastic signals[J]. Geoscience and Remote Sensing, IEEE Transactions on, 2014, 52(4): 2261-2277.

[3] Guo Y, Wang D, He X, et al. Super-resolution imaging method based on random radiation radar array[C]// Imaging Systems and Techniques (IST), 2012 IEEE International Conference on. IEEE, 2012:1-6.

[4] Wang Dongjin, Meng Qingquan, Liu Falin, et al. Microwave staring correlated imaging random radiation array element spatial arrangement optimization method. CN: 104597445A, 2015-05-06.

[5] Li Jun, Li Xiaomin, Zhu Shengqi, et al. Microwave correlated imaging method based on radar rotating emission array.CN: 104199028A, 2014-12-10. 